\title{
LA OUESTIONE DELLA LINGUA ITALIANA ATTRAVERSO I SECOLI
}

\author{
Carolina Massi Albanese \\ Universidade Federal do Paraná \\ Luciana Albanese \\ Mestranda na USP
}

\begin{abstract}
RIASSUNTO
Abbiamo ripercorso, rapidamente, l'itinerario linguistico, dai primi documenti in "volgare" ai nostri giorni, tenendo conto dei fatti di lingua più efficaci per l'incremento dell'italiano in un ambito nazionale.
\end{abstract}

\section{A. PRIMI DOCUMENTI DEL VOLGARE ITALIANO.}

L'unità del latino medievale rispecchiava un'unità storicocivile che comprendeva gran parte dell'Europa ed aveva un suo proprio nome: Romania. Con questo nome s'indicavano i paesi latini raggruppati sotto il segno di Roma ed essi se ne facevano un vanto in antitesi con la barbarie dei non latini. Questa funzione polemica rimase costante nel "romanice loqui" contrapposto al "barbarice loqui".

Non si può praticamente parlare di una transformazione, mê piuttosto di molteplici insensibili e sucessive trasformazioni, che noi possiamo cogliere solo in parte nei documenti e che, sommate insieme, distinguono dalla latina quella che noi chiamiamo "lingua italiana".

Nel secolo VIII si può dire che tale processo sia ormai concluso: non ne fu estranea la Chiesa che, pur essendo la custode dell'antico sapere e dell'antica letteratura, sentì la necessità di un rapporto più stretto con il popolo che per la maggior parte non comprendeva più il latino. Si tiene a Tours nell'813 un concilio che prescrive ai Vescovi di tenere dall'ora in poi le prediche secondo il dialetto dei loro fedeli, e cioè di tradurre le loro omelie dal latino in "rustica romana lingua 
aut teotisca", vale a dire nel gallo-romano o in tedesco, poiché l'impero carolingio era appunto costituito di popolazioni romane e tedesche.

Proprio in Francia abbiamo il 1." documento ufficiale in volgare neolatino sono i Giuramenti di Strasburgo. In Italia, una sicura testimonianza dell'uso pubblico del volgare appartiene a circa un secolo dopo e ci è data dal Panegirico dell'imperatore Berengario, in una cerimonia avvenuta nel 915 a Roma. Si dovrà lasciar passare del tempo per trovare un'altra testimonianza dell'uso del volgare nella predicazio. ne: infatti l'epitaffio sulla tomba del papa Gregorio V ci fa conoscere che questo pontefice era solito rivolgersi al popolo in tre diverse lingue: la francese, la volgare e la latina. Soltanto nel XIII secolo appariranno i primi testi in volgare di una certa importanza letteraria: per ora si tratterà di usare il volgare in scritture di carattere eminentemente pratico. Il più antico testo in cui comunemente, ma non senza discussioni, si ritiene di poter ravvisare manifestati i tratti di volgare sintatticamente regolare, è un indovinello veronese del secolo IX scoperto nel 1924 da Luigi Schiaparelli in un codice della Biblioteca Capitolare di Verona: "Se pareba boves, alba pratalia araba/albo versorio teneba, negro semen seminaba". L'indovinello, derivato da una metafora antichissima, significa il confronto fra l'aratura e la scrittura. Bruno Migliorini' ${ }^{1}$ osserva che l'indovinello risale all'epoca di Carlo Magno e che, pur non accettando la tesi di quelli che fanno persistere il "latino volgare" fino a quest'età, si deve riconoscere la difficoltà di stabilire la differenza tra la lingua parlata, ricca già di innovazioni tipiche della lingua nuova, e la lingua scritta "in cui i chierici incoltissimi introducono sporadicamente i loro volgarismi".

Le formule dei cosiddetti "Placiti cassinesi" hanno un pieno carattere di documento del nuovo volgare, per la prima volta consapevolmente opposto al latino: la loro datazione risale al 960 e al 963 . Si tratta di quattro pergamene di analogo argomento, e precisamente di tre placiti ed un memoratorio sull'apartenenza di certe terre il cui possesso era rivendicato nella persona dell'abate o del preposto della celeberrima Abbazia di Montecassino o di monasteri da essa dipendenti. Il fatto è che, nel dispositivo delle sentenze, redatto normalmente in latino secondo la consuetudine dei tempi, le formule testimoniali sono inserite nella lingua volgare nella quale appaiono essere state pronunciate. Pare legittima la supposizione che si sarà ritenuto opportuno far intendere la testimonianza in maniera inequivocabile da tutti i presenti

1 MIglioriNi. B. Storia della lingua italiana. Firense, Sansoni, 1962 p.62 
al giudizio, compresi gli ignari di latino, così come nei giuramenti di Strasburgo.

Senza volerci dilungare sui primi documenti del volgare italiano, possiamo evidenziare che nel XII secolo si è ormai delineato il quadro dell'Italia dialettale in cui emergono Roma e Montecassino come centri di maggiore influenza: il volgare si è affermato come pratico strumento linguistico, mentre il latino continua a vivere indisturbato come lingua letteraria.

\section{B. IL PRIMO PADRE DELLA LINGUA ITALIANA: DANTE ALIGHIERI.}

I primi documenti in volgare manifestano la lenta $e$ graduale infiltrazione dei parlari dialettali nelle iscrizioni, nelle trascrizioni giuridiche e religiose, nei documenti scritti. Questo fenomeno rimaneva però collegato all'unità contingente di farsi intendere dai testimoni o dai contraenti di un atto notarile, di riprodurre fedelmente le confessioni dei fedeli, ecc.

Il problema di utilizzare la parlata romanza a scopi puramente letterari è stato sollevato per la prima volta da Dante Alighieri (1265-1321): il poeta fiorentino non ha solamente il merito di averci lasciato nella Divina Commedia la prima testimonianza di un'opera completamente organica in volgare, $\mathrm{ma}$ anche quello di aver codificato il suo pensiero sulla lingua in uno scritto teorico che ci rivela con quanta preparazione e con quali motivazioni linguistiche egli si sia accostato alla composizione del suo poema.

Il De Vulgari Eloquentia fu scritto nei primi anni del primo decennio del XIV secolo e verosimilmente tra il 1303 e il 1305. Allo stesso periodo risale la stesura del Convivio il cui primo libro tratta diffusamente di questioni linguistiche. Tra gli ultimi decenni del XIII e la prima metà del XIV secolo si diffonde in Europa, insieme all'averroismo (o aristotelismo radicale), una nuova teoria linguistica, la cosiddetta grammatica modista o speculativa. Le due opere filosoficamente più significative appartengono a due danesi: i Modi Significandi sive Quaestiones super Priscianum Maiorem di Boezio di Dacia e i Modi Significandi di Martino di Dacia. Maria Corti' richiama l'attenzione degli studiosi sulla relazione tra alcuni temi del De Vulgari Eloquentia e i Modi significandi di Boezio. Il termine 'grammatica' copre in Boezio tre differenti realtà, anche se tra loro strettamente legate:

2 CORTI, M. 'La teoria del segno nei lociei modisti c in Dante'. Quardeni del circolo Semiologico Siciliano 115i:69-86: mar. 1981 
1. Grammatica come struttura mentale "naturaliter ab homine habita". Il parlare e la grammatica profonda che lo regola non sono acquisiti per imitazione ma sono l'effetto necessario delle capacità naturali dell'uomo.

2. Grammatica come teoria o "inventio philosophiae". L'argomento conclusivo di Boezio è che "inventio grammaticae praecedit ipsam grammaticam. Ideo qui invenit grammaticam non fuit grammaticus.": Solo al filosofo spetta il privilegio della "inventio grammaticae".

3. Grammatica particolare di una lingua particolare. $E$ questo il dominio proprio del grammatico empirico illuminato dai principi della filosofia. Il grammatico, diversamente dal filosofo, non inventa i "principia grammaticae" ma, supponendoli li applica allo studio di lingue specifiche.

L'accezione predominante di "gramatica" è formulata nel De Vulgari Eloquentia di Dante e ricorre spesso anche nel Convivio. La distinzione tra idiomi-volgari e idiomi-grammatiche è introdatta nella prima pagina del De Vulgari Eloquentia. ${ }^{*}$ La lingua volgare è il linguaggio che si apprende senza norme, imitando la nutrice, e la grammatica cioè il linguaggio letterario che, ai suoi tempi, è il latino codificato ormai in un rigido sistema grammaticale, che si apprende con lo studio ed è governato dall'arte. Quindi, volgare rappresenta il polo della natura, "gramatica" quello della "ars" o cultura: il primo "naturalis est nobis", la seconda "potius artificialis". Dei primi due, il volgare è il più nobile perchè più naturale.

Le differenze rispetto alla linguistica boeziana sono no. tevoli. La grammatica in Boezio è contemporaneamente una realtà naturale-esiste "ab aeterno" nella mente umana-ed una raffigurazione filosofica. In Dante, gli idiomi-volgari sono naturali e universali; le grammatiche sono idiomi ausiliari artificialmente costruiti che si aggiungono ai primi.

Dante rivolge il suo esame all'italiano passando alle parlate italiane che classifica in quattordici gruppi principali, sette a oriente, sette a occidente degli Appennini. Scarta i dialetti più rozzi e primitivi, quali il romano (pretenzioso e reboante), il marchigiano, lo spoletano e il milanese (troppo presuntuosi), il rude friulano, il sardo pedissequo imitatore del latino, il genovese, il romagnolo ed il veneto. Elogia il siciliano, ma quello illustre, usato dai rimatori della scuola di Federico II. Nessuno dei dialetti scelti, in quanto tali, raggiunge la dignità di lingua nazionale, nonostante che in tutti si possa trovare come un presagio di essa.

4 ALIOHIERI, D. De Vulgari Eloquentia. Padora. Anterore. 1068. 
In realtà mancavano le condizioni storiche e politiche perché una lingua del genere potesse formarsi senza difficoltà. La minuta suddivisione politica e l'assenza di una corte (aula) impedivano agl'intellettuali di riunirsi ed elaborare una lingua non più particolare di una regione.

Il volgare ideale, per Dante sarebbe "unitario" e organico rispetto alla frantumazione dei dialetti; "illustre", perché può dare gloria illustrando chi lo sappia usare; "cardinale", perché attorno ad esso devono ruotare come attorno a un cardine, tutte le altre parlate municipali; "aulico" perché se l'Italia avesse una corte (=aula) sarebbe il solo degno di essere parlato in essa; "curiale", perché curialità significa la norma dell'agire umano secondo la ragione e la legge quali si attuano nella Curia.

Nell'altra opera teorica di Dante, il Convivio, sono spiegate le ragioni che lo hanno spinto a redigere in volgare e non in latino. La prima motivazione è tecnica ed artistica per la correlazione ed interdipendenza delle canzoni composte in volgare, la seconda motivazione è data dal suo desiderio di raggiungere, con un eguale veicolo linguistico, il maggior numero di persone, la terra motivazione è il naturale amore della propria loquela. Dante chiude il Convivio con un tono profetico: "Questo sarà luce nuova, sole nuovo, lo quale surgerà là dove l'usato tramonterà, e darà lume a coloro che sono in tenebre e in oscuridade, per lo usato sole che a loro non luce!"

\section{LE VICENDE DEL "VOLGARE" NEL QUATTROCENTO.}

Dopo il fenomeno non indifferente della spinta linguistica data dai tre grandi scrittori: Dante, Petrarca e Boccaccio, la ricerca di una lingua si arena nelle vicende storico-sociali della Penisola. La lirica e la prosa in volgare vengono considerate generalmente "passatempi" diffusi tra la borghesia cittadina in Toscana o alle corti settentrionali. Il volgare, ban. dito da tutti gli usi di grande impegno, occupa invece uno spazio sempre maggiore negli usi pratici.

Il progressivo riemergere del volgare, a scapito del latino umanistico, verso la fine del Quattrocento, fu determinato dall'intensa ed animata vita sociale nelle corti e dai rapporti sempre più intensi fra le regioni italiane.

Naturale, quindi, che si riproponesse la "questione della lingua", cioè che si dibattesse il problema di come uscire dai particolarisimi regionali per formare una lingua letteraria nazionale. 
Secondo Giacomo Devoto,' i principi sui quali s'imper. niarono le discussioni furono principalmente quattro:

1. Si discusse della superiorità del latino o del volgare in base all'ambito e cioè allo spazio nel quale esse potevano essere validamente impiegate.

2. Un secondo criterio era quello del pregio intrinseco. A questo proposito Leon Battista Alberti aveva sostenuto che la perfezione di una lingua sta non nella sua materia, ma nel suo uso.

3. Terzo criterio fu quello dell'autonomia, cioè il pregiudizio che l'italiano fosse una "corruzione" del latino. La giusta risposta fu data da Benedetto Varchi, il quale scrisse che il volgare è una lingua nuova: "non corruzione ma generazione".

4. Ultimo criterio è quello della regolarità, nel quale il latino è fermo, mentre il volgare è mutevole, o dantescamente, è un "uso".

Finché si poté credere che la nuova civiltà fosse un ri. torno agli antichi, era naturale, scrive il Migliorini, ${ }^{6}$ che il linguaggio di Roma paresse il suo unico mezzo di espressione, ma quando si cominciò ad acquistare coscienza che nella nuova cultura si esprimeva l'anima moderna, allora il volgare risorse come il linguaggio del nuovo spirito.

Ad indizio della resurrezione del volgare si pone un episodio letterario fiorentino del 1441. In quell'anno Leon Battista Alberti promesse una gara sovvenuta da Pietro de'Me. dici, bandita solennemente dagli Officiali dello Studio, celebrata nella Chiera di S. Maria del Fiore, a Firenze. Era una gara poetica sul tema della "vera amicizia", la quale poiché al vincitore era posta come premio una corona d'argento, si disse Certame coronario. La gara si concluse senza vincitori, ma l'episodio è significativo ad indicare l'acquisita coscienza delle possibilità del volgare. L'iniziativa può assere assunta come data d'inizio della lingua letteraria italiana.

Nei primi decenni del secolo XVI si ebbero accese dispute intorno alla lingua per fissare il lessico e la grammatica, determinando il tipo di lingua unica per tutti gli scrittori d'Italia. Infatti, anche se vi erano state le opere dei grandi trecentisti, nonché quelle del Boiardo e del Sannazzaro, nel secolo XV, in tutte le sfere sociali e persino tra gli scrittori, si usavano, per lo più, le parlate locali. Chi affrontò la questione di una lingua unitaria e propose una soluzione che rimase salda per tre secoli, fu Pietro Bembo. 
Nelle 'Prose della volgar lingua il Bembo sostiene la piena dignità culturale della lingua italiana. Se si fa eccezione per le Regole della volgar lingua teorizzate dal Fortunio, de Prose del Bembo possono essere considerate la "prima grammatica della lingua italiana". Il Bembo proclama l'eccellenza del fiorentino (non già quello parlato, ma quello elaborato dai grandi trecentisti: Petrarca per la poesia, Boccaccio per la prosa) su tutti gli altri volgari. In questo modo veniva sancito, anche per il volgare, il principio dell'imitazione dei modelli scritti. Il Bembo escludeva Dante, ritenuto nella sua mirabile ricchezza di stile, troppo realistico e violento.

Alla teoria del Bembo si opposero altri letterati del tem. po, dando così inizio ad una nuova questione della lingua. Alla corrente arcaicizzante del Bembo, si ribellano quella di tipo eclettico, ispirata alla coiné delle corti (Vincenzo Colli, Angelo Colocci, Giangiorgio Trissino, ecc.) e quella dei sostenitori del fiorentino parlato (Machiavelli, Giovambattista Gelli, Pier Francesco Giambullari, ecc.).

L'impostazione del Bembo è eminentemente retorica ed è facile, a secoli di distanza, afferma Mario Sansonei, indicarne i punti deboli: a) la concezione di una lingua che debba riportarsi ai trecentisti, pertanto di una lingua rigida ed immobile; b) il concetto d'imitazione; c) l'esagerato fiorentinismo. Eppure, sostiene lo studioso, la dottrina bembesca offriva la soluzione più sensata per il bisogno della cultura italiana. Il Bembo infatti determinava un punto di riferimento per tutti gli scrittori, additava, rifacendosi al Petrarca ed al Boccaccio il piu alto livello della lingua italiana, ed impediva una dispersione municipalistica della lingua italiana letteraria.

\section{L'ACCADEMIA DELLA GRUSCA.}

Cosimo I de'Medici, granduca di Toscana, va ricordato per i suoi tenaci tentativi compiuti allo scopo di promuovere lo studio e la codificazione della lingua con il compito di ridurre ogni scienza nel volgare toscano. A tal fine egli aveva riconosciuto ufficialmente, con un decreto, l'Accademia fiorentina e le aveva affidato il compito di fissare per iscritto le regole della lingua. I suoi desideri di mecenate non furono realizzati dall'Accademia che aveva creato, ma furono invece soddisfatti in parte da un'altra che era nata come libero sodalizio di alcuni componenti della brigata dei "Buontemponi", con l'intenzione di contrapporsi alla pedanteria 
dell'Accademia fiorentina. Tale Accademia nacque Ufficialmente nel 1583, suprattutto per opera di Leonardo Salviati, ed ebbe il nome di Accademia della Crusca, mentre i suoi componenti passarono ad essere "Crusconi" invece che "Buontemponi": uomini addetti a vagliare la farina e separarla dalla "crusca". Per tal motivo, insegna dell'Accademia fu il frullone col motto: "Il più bel fior ne coglie", ricavato da un verso del Petrarca. L'Accademia si propose la preparazione di un vocabolario concepito come tesoro della lingua e che registrasse tuttte le parole dell' uso fiosentino trecentesco attestato dagli autori maggiori e minori in scritture private e pubbliche.

Il Vocabolario degli Accademici della Crusca uscì a Venezia nel gennaio del 1612. Il rigore applicato nel preparare il Vocabolario era lo stesso che aveva portato Leonardo Salviati a condannare il Tasso per le sue innovazioni linguistiche ed a pregiare l'Ariosto per la sua misura classica anche nella lingua. Il vocabolario registrava infatti, in appendice, le forme accettabili attinte da testi del Cinquecento. Tanta intransigen. za suscitò subito la reazione di alcuni, tra i quali il Tassoni, cosicché gli Accademici credettero opportuno allargare lo spoglio ad altri autori nella seconda edizione veneziana. Una profonda transformazione si ebbe, vari decenni dopo, con la terza edizione di Firenze, nel 1691, ampliata da uno a tre volumi, e che accettava anche la lingua del Tasso.

L'eco del Vocabolario si fece sentire fortemente pure all'estero, divenendo il capostipite dei dizionari delle lingue moderne. Con la quarta edizione in sei volumi, stampati a Firenze nei primi decenni del Settecento, si chiude il primo periodo di attività dell'Accademia della Crusca.

\section{E. IL SECONDO PADRE DELLA LINGUA ITALIANA: ALESSANDRO MANZONI.}

L'affermazione del "volgare", cioè della lingua parlata, in Italia, era stata particolarmente difficile e contrastata dal Latino, rimasto fino al Seicentto la lingua della Chiesa, dell'Amministrazione e della Giustizia. Anche quando ebbe finalmente vinto la sua battaglia, l'italiano restò, rispetto al latino, in una posizione subalterna e come afflitto da un complesso d'inferiorità nei suoi confronti: veniva infatti insegnato secondo le regole del latino. Per di più, Paese policentrico, l'Italia non aveva mai avuto una capitale come $\mathrm{Pa}$ rigi, che dava il "là" a tutto, anche alla lingua, dettandone il modello al resto della Francia. Gli intellettuali che avrebbero 
dovuto assumersene il compito erano sparpagliati nelle Corti dei vari Comuni, Signorie e Principati, ognuna delle quali aveva un suo gergo. $\mathrm{Ma}$, oltre a questo, erano mancate le palestre. Il francese aveva avuto i "salotti", dove cultura e società s'incontravano facendo della lingua colta una lingua di conversazione e della lingua di conversazione una lingua colta.

L'inglese aveva avuto il Parlamento e i 'clubs', ed era questo che lo rendeva cosi concreto e pratico. Gl'Italiani non avevano avuto che l'Accademia dove il dotto parlava al dotto in una lingua convenzionale, che non aveva più nulla a che fare con quello dell'uso comune e che cercava di somigliare al latino.

Il Vocabolario della Crusca, iniziato nel 1612 e proseguito fra roventi polemiche e contestazioni, dopo duecent'anni non era ancora arrivato a termine. Era un dizionario che accoglieve i termini più arcaici e in cisuso, rifiutando tutti gli apporti della lingua vera, quella che si parlava nelle strade e nelle piazze. Questo divorzio fra le due lingue non era che il riflesso di quello, sempre esistito, fra cultura e società.

Nel Settecento la disputa principale è tra i fautori e gli avversari dello "scriver toscano" (cioè del toscano trecentesco) che era codificato nel Vocabolario della Crusca, uscito nella sua quarta edizione. L'arcaico toscaneggiante è oppugnato fra i più intelligenti letterati del secolo, dal Mascheroni al Baretti. L'opposizione più radicale viene da Alessandro Verri che nella rivista Caffé fa la sua solenne rinunzia alla pretesa purezza della toscana favella.

Larga eco ebbe il Saggio sopra la lingua italiana di Melchiorre Cesarotti, ristampato poi nel 1800 con il nuovo titolo di Saggio sulla filosofia delle lingue nel quale si mirava a romper certi pregiudizi ed a rendere la lingua saggiamente libera. Cesarotti fu principalmente criticato per il suo atteggiamento rispetto al francesismo, per l'illustrazione dei principi secondo i quali un popolo che riceve da un altro, alimenti di pensiero, ne riceve anche di parole.

L'uscita della nuova edizione del Vocabolario della Crusca aumenta le dispute fra partigiani ed avversari, finché Pietro Leopoldo, nel luglio del 1783 sopprime l'accademia della Crusca, fondendola con l'Accademia Fiorentina e con quella degli Apatisti, sotto l'unico nome di Accademia Fiorentina.

L'abate nizzardo Francesco D'Alberti di Villanova, il quale aveva compilato un dizionario francese-italiano e viceversa, riusciva a portare a compimento un Dizionario universale critico-enciclopedico in cui sono incluse numerose voci scientifiche e di arti e mestieri. 
L'influenza inglese, non cosi ampia come quella del francese, è dovuta all'ammirazione per la letteratura, filosofia, scienze, ed ai lunghi soggiorni di italiani in Inghilterra, come il Rezzonico e l'Alfieri.

Le discussioni sulla lingua diventarono particolarmente aspre fra la fine del Settecento ed i primi dell'Ottocento. Lo spunto fu dovuto alla nascita dell'Istituto nazionale di Scienze, Lettere ed Arti che, fondato da Napoleone a Milano, come corrispettivo italiano dell'Accademia di Francia, si proponeva fra gli altri compiti, anche quello di mettere ordine alla lingua. I letterati si divisero: da una parte i cosiddetti puristi, fedeli alla Crusca, e capitanati dall'abate veronese Cesari, secondo il quale la lingua italiana era quella degli scrittori del Trecento, e tutto ciò che era avvenuto dopo era da buttar via; dall'altra, gl'innovatori capitanati dal Monti, il quale pretendeva innovare adottando non la lingua successiva al Trecento, ma quella precedente, cioè quell'italiano illustre che diceva, era stato patrimonio di tutto il Paese e non monopolio della Toscana. In realtà Monti intendeva che la lingua dovesse restare una lingua non di popolo e per il popolo, ma di dotti per dotti, fabricata in 'aula' ed imposta dalla cattedra: che era la posizione tipica del letterato 'cortigiano' come lui, al servizio non del 'pubblico', ma della 'casta'.

Queste pedantesche risse dimostrano quanto lontani fossero i letterati italiani da ogni concezione d'impegno civile: invece di affrettarla, ritardavano la nascita di una lingua italiana che tutti gl'italiani potessero scrivere come si parlava e parlare come si scrivera.

I sogni dei patrioti romantici si attuano mentre opera uno dei maggiori romanzieri italiani: Alessandro Manzoni. Il Manzoni si ricollega in molti sensi al Porta: anzitutto perché egli accetta in partenza l'ampliamento dell'orizzonte civile proposto dal Porta, facendo protagonisti del suo romanzo un lavoratore ed una contadina, e muovendo intorno ad essi tutta una società stratificata, poi perché questo ampliamento corrisponde ad una interiore rivendicazione della nobilità e della libertà di ogni creatura umana. Ma soprattutto perché il Manzoni riprende, con rinnovato vigore e rigore, le meditazioni linguistiche del Settecento, portandole ad una conclusione diversa: e cioè dopo il tentativo di ricuperare il dialetto milanese come supporto per una lingua italiana, la decisione di accogliere il toscano. Si badi, non il toscano della convenzione letteraria, ma il toscano vivo. Soluzione audace che permetteva di raggiungere un linguaggio comune (preoccupazione dell'unità nazionale anche linguistica) perché simile a quello letterario (toscano antico) ma, a differenza di quello, 
duttile, pronto al dialogo, non compromesso da convenzioni accademiche. In altre parole, il Manzoni riprende i tentativi del Goldoni e del Porta, ma li riprende al cospetto di una unità nazionale ed ideale. Alla luce della soluzione manzoniana, il ricorso ai dialetti appare come una fase interlocutoria nella ricerca di una lingua che sappia esprimere completamente la nuova realtà sociale e nazionale.

La riflessione sulla lingua ha accompagnato il Manzoni per tutta la sua vita: già si parla di lingua nella lettera al Fauriel del 9 febbraio 1806, se ne parlerà al Casanova nel 1871. Un iter meditativo linguistico lunghissimo, con mutamenti d'orizzonte. Come in Dante e nel Machiavelli, c'è nel Manzoni il continuo desiderio di controllo della realtà linguistica del proprio tempo, dunque, una profonda coscienza sociale.

Dove trovarla, confidava il Manzoni al Flauriel, una lingua italiana semplice, piana, discorsiva, che tutti potessero capire ${ }^{8}$ La sua prima idea fu di scrivere il suo romanzo in francese e fu lo stesso Fauriel a sconsigliarlo per fortuna sua e nostra. Fu un autentico tormento di cui offrirono una patetica testimonianza i tribolatissimi manoscritti e le minute postille. Finita la prima stesura, essa gli parve infiorata di tali e tanti modi gergali lombardi, che fu colto dalla tentazione di disfare tutto e di rifarlo in dialetto. Ancora una volta fu il Fauriel a sconsigliarlo. Ma il problema restava: dove trovarla quella benedetta lingua, che fosse insieme tanto corretta e popolaresca da poter stare nei dialoghi, tanto sulla bocca del Cardinale Borromeo che su quella del contadino Renzo? Fu allora che il Manzoni pensò di "sciacquare i panni in Arno", cioè di andare a cercarsi quella lingua in Firenze. Ciò che Firenze offriva e offrì al ,Manzoni era il modello di una lingua che aveva abolito, o per lo meno di gran lungo ridotto, il divario fra il vocabolario delle persone colte e quello del popolo. Di questi risultati a noi interessa soprattutto uno: gl'italiani finalmente avevano un libro che tutti coloro che sapevano leggere potevano leggere ed in cui trovavano un punto di fusione, fin allora mai raggiunto, fra la lingua parlata e quella scritta. Ma il libro aveva anche un altro grande merito: quello di essersi dato a protagonista non piì l'Eroe, il personaggio di eccezione, ma il popolo, il vero popolo. Per la prima volta il comune lettore che la letteratura aulica e cortigiana aveva sdegnosamente escluso, riconosceva se stesso ed i propri simili negli attori di una vicenda, di cui in tal modo si sentiva partecipe. Ecco perché i Promessi Sposi

8 CECCHI, E. Ritratti e Profili. Milano, Rizzoli, 1954 p.125.

9 CECCHI. D.130. 
furono il più grande evento di questo periodo, e non soltanto sul piano letterario.

Come scrive il Migliorini ${ }^{10}$, la grande innovazione manzoniana consiste nel transformare una disputa di letterati in un problema civile che riguarda tutta l'Italia.

E per la lingua Manzoni combattè molte battaglie non scevre d'amarezza. Il ministro della Publica Istruzione, Broglio, aveva nominato una Commissione con l'ufficio preciso di ricercare e di proporre tutti i provvedimenti coi quali si potesse aiutare a rendere piì universale la conoscenza della buona lingua e della buona pronuncia. Manzoni accettò di parteciparvi e di esserne il presidente. La Commissione si divi. deva in due sezioni: di quella milanese facevano parte iI Manzoni, il Carcano e il Bonghi; dell'altra fiorentina, il Lambruschini, Tommasini ed altri. La relazione del Manzoni intitolata "Del'unità della lingua e dei mezzi per diffonderla", pubblicata sulla Nuova Antologia di Firenze, riassumeva tutto il suo pensiero al riguardo. In Firenze si trovano tutte le cognizioni, le opinioni ed i concetti d'ogni genere che ci possano essere in Italia, e che, d'altronde, sosteneva il Manzoni, non esiste in Italia una lingua comune di fatto, ma solo una quantità indefinita di locuzioni comuni. Non si tratta, secondo il Manzoni, tanto di una questione teorica, quanto di un problema politico, perché, dopo l'unità di governo, d'armi e di leggi, l'unità della lingua è quella che serve il più a rendere strette l'unità di una nazione."

Il Lambruschini partiva da presupposti affatto diversi dal Manzoni; per lui la lingua italiana era quella consacrata dall'uso degli scrittori: si trattava quindi di formare il vocabolario su quell'uso e non sul dialetto di Firenze. Anche se il Lambruschini si guardasse bene dal mettersi contro il Manzoni, questi s'avvide benissimo della diversità del pensiero, rassegnò le dimissioni dalla Presidenza continuando ad elaborare la nuova Appendicc alla Relazione. Nell'Appendice e poi nella lettera al Casanova, il Manzoni con abbondanza di argomenti ripeté quello che aveva avuto occasione di dire e di scrivere mille volte, ossia che posto che 1'Uso sia la propria materia di un Vocabolario, ne segue che il criterio per sceglierne i materiali non possa essere senz'altro che l'uso medesimo. ${ }^{12}$

\section{F. G.I. ASCOLI E L'ITALIANO DALLUNITA D'ITALIA AI GIORNI NOSTRI.}


Il Manzoni aveva trovato una realtà idiomatica, la lingua viva parlata dalle persone colte di Firenze, dunque una norma valida insieme per gli scrittori e per i parlanti italiani. Egli aveva finalmente raggiunto il triangolo tanto auspicato: lingua viva, lingua letteraria, stile dello scrittore. ${ }^{13}$ Triangolo funzionalissimo per lui scrittore, ma non altrettanto funzionale per g'italiani, come la storia dimostrò. Primo ad averne chiara coscienza fu l'Ascoli. Contrapponendo al criterio normativo manzoniano una visione storica, l'Ascoli sostenne che non si potevano sacrificare le varietà linguistiche regionali sovrapponendo loro un modello toscano imposto dall'alto; affermò inoltre che l'unificazione linguistica italiana sarebbe stata solo una conseguenza di un espandersi della cultura attraverso tutta la penisola, in senso non solo orizzontale, ma verticale, cioè con la sua penetrazione negli strati più popolari del Paese. ${ }^{1+}$ Questa verità nulla toglie tuttavia alla grandezza del Manzoni: mirabile da un lato l'aspirazione a conquistare alla letteratura nuove classi di lettori, e splendido il progetto di dare agl'italiani una lingua unitaria.

Rimandiamo al nostro precedente articolo sulla comunicazione e gli usi della lingua di oggi. ${ }^{15}$ La divulgazione della stampa ha reso sensibilmente uniforme la lingua scritta. La scuola, che impone un linguaggio criticabile in quanto pedan. tesco, ma che è comunque unitario, in particolare per quanto riguarda il piano dell'espressione.

Quale sia per essere la lingua di domani, non è possibile vaticinare se non ripetendo quelle parole con cui Gino Capponi concludeva il uso saggio della Nuova Antologia (1869): "la lingua italiana sarà ciò che sapranno essere gli Italiani"..6

\section{RESUMO}

Percorremos rápido o itinerário linguístico, desde os primeiros documentos em 'vulgar' até os dias atuais, levando em consideração os fatos de língua mais eficazes para o incremento do italiano no âmbito nacional.

\section{RIFERENZE BIBLIOGRAFICHE}

ALBANESE, C. La comunicazione e gli usi della lingua italiana di oggi. LETRAS (34):3-12 85 UFPR.

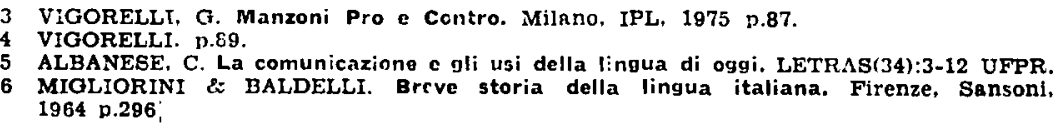


ALBANO \& LEONI. Italia linguistica. Bologna, Il Mulino, 1963. 376p. ALIGHIERI, Dante. De Vulgari Eloquentia. Padova, Antenore, 1968. CECCHI, E. Ritratti e Profili. Milano, Rizzoli, 1954. 292p.

CORTI, M. 'La teoria del segno dei logici modisti e in Dante'Quaderni del Circolo Semiologico Sichliano(15):69-93; mar. 1981. DEVOTO, G. Il linguaggio d'Italia. Milano, Rizzoli, 1972. 407p. MIGLIORINI, B. Storia della lingua italiana. Firenze, Sansoni, 1962. $708 \mathrm{p}$.

MIGLIORINI \& BALDELLI. Breve storia della lingua italiana. Firenze, Sansoni, 1964. 372p.

SANSONE, M. Storia della letteratura italiana. Torino, Principato, 1949. 310p.

VIGORELLI, G. Manzoni Pro e Contro. Milano, IPL, 1975, 980p. 